\title{
Incident Occurrence Modeling during Hurricane Evacuation Events: The Case of Alabama's I-65 Corridor
}

\author{
Daniel J. Fonseca, ${ }^{1}$ Yingyan Lou, ${ }^{2}$ Gary P. Moynihan, ${ }^{3}$ and Saravanan Gurupackiam ${ }^{4}$ \\ ${ }^{1}$ Department of Mechanical Engineering, The University of Alabama, Tuscaloosa, AL 35487, USA \\ ${ }^{2}$ School of Sustainable Engineering and Built Environment, Arizona State University, Tempe, AZ 85281, USA \\ ${ }^{3}$ Department of Civil, Construction, and Environmental Engineering, The University of Alabama, Tuscaloosa, AL 35487, USA \\ ${ }^{4}$ Penn State University, Harrisburg, Middletown, PA 17057, USA \\ Correspondence should be addressed to Daniel J. Fonseca; dfonseca@eng.ua.edu
}

Received 28 June 2013; Accepted 19 September 2013

Academic Editor: Luis Carlos Rabelo

Copyright ( 2013 Daniel J. Fonseca et al. This is an open access article distributed under the Creative Commons Attribution License, which permits unrestricted use, distribution, and reproduction in any medium, provided the original work is properly cited.

\begin{abstract}
Contraflow on major evacuation routes is one scheme that has been adopted in many Gulf and eastern coastal states for hurricane evacuation. The idea is to reverse one direction of the roadway in order to accommodate the often substantially increased travel demand moving away from the impact area. Efficient planning and operation is critical to a successful contraflow implementation. Alabama has an approximately 140-mile contraflow segment on I-65 between exit 31 and exit 167 and has carried out contraflow operations several times in past hurricane evacuations. The timing for the deployment of equipment and personnel and the initiation and termination of actual contraflow affects the effectiveness, safety, and cost of the operation. Researchers from the University of Alabama were tasked with the design of a decision support system for contraflow evacuation planning. The conceived decision support system consists of three main modules: the demand module, the network optimization module, and the incident and characterization module. This paper focuses on the design of the traffic incident generation and characterization module of the planned decision support system.
\end{abstract}

\section{Introduction}

Massive evacuations due to tropical storms, hurricanes, and other extreme weather events are becoming routine events in coastal regions around the globe. The normal response to these occurrences is to evacuate inland from the coast. Normal traffic flows turn into congestion, frustration, and gridlock. This reduces the number of vehicles that can leave the affected area if an evacuation order is given. The potential risk for catastrophic loss increases if the hurricane strikes stalled traffic and necessitates further evacuation planning. Among the most common evacuation strategies flowed by traffic management officials during such events is "reverselaning" or "contraflow." Contraflow is the process of reversing one direction of traffic on specific routes to facilitate overall evacuation flow. This procedure is generally applicable to interstates, referred to as "denied access routes," since traffic control can be applied to interchanges and terminal areas.
During contraflow operations an increase of vehicular traffic incidents should be expected. Traffic incidents are defined as nonrecurring events, including accidents, that lead to the overcongestion or saturation of a network of roads. During large-scale evacuations such as in the case of a hurricane evacuation, traffic incidents significantly extend the travel time of evacuees; thus, the time needed to completely empty the region under hazard increases marginally.

The authors of this study were tasked by the Alabama Department of Transportation (ALDOT) to develop a decision support system for contraflow planning during hurricane evacuation. Alabama and several other neighboring states have adopted the concept of contraflow in hurricane evacuation. In Alabama, an approximately 140 -mile section of I- 65 between exit 31 and exit 167 is identified as the contraflow segment. In this case, I-65 would be reverse-laned such that all traffic would flow north, from south of Alabama Route 225 , near Mobile, to exit $167 / 168$, just south of the greater 
Montgomery metropolitan area [1]. As noted in Figure 1 [1], this concept was further refined after Hurricane Katrina, to provide emergency vehicles with an alternate route south via U.S. 31.

Although the contraflow operation increases roadway capacity for evacuation, it is by nature an unusual measure. Practical implementation issues range from traffic control, access management, and use of roadside facilities to safety, labor requirements, and cost [2]. Therefore, care must be taken in the planning and real-time operations of contraflow evacuation. A design for a computer tool to help the decision making in contraflow scheduling was thus developed by the researchers to provide ALDOT with (1) a well-established and reliable mesoscopic traffic flow simulation, namely, the cell transmission model; (2) a sensitivity analysis to account for inaccurate route choice and incident information; (3) a robust and stochastic optimization platform to determine the onset and duration of contraflow; and (4) an assessment of the impact that traffic incidents may have in the overall evacuation effort. Figure 2 depicts the architecture of such a decision support system (DSS). This paper discusses the development and validation of the incident generation and characterization module.

\section{Background Studies}

The currently available literature provides evidence of the difficulty in addressing the impact of potential traffic incidents during massive evacuation events, especially under contraflow and when the weather conditions are deteriorating. There is just a handful of published studies that deal with the generation and characterization of random traffic incidents during hurricane evacuations.

Lindell and Prater [3] conducted a study that lists the principal behavioral factors affecting hurricane evacuation time estimates (ETEs). Although, among these factors, the authors mention the impact that traffic incidents originated from hazardous conditions such as storm surge and wind has on evacuees' travel times and route selections; accessing the role of incidents in hurricane evacuations was outside the scope of their investigation.

Wolshon et al. [4] reviewed traffic operations, management, and control during hurricane evacuation, including reserve-laning practices. According to the authors, one of the primary considerations while deciding on contraflow measures is safety, as reversing the movement of traffic increases the likelihood for sideswipe and head-on collisions. Wolshon et al. concluded that during hurricane evacuations, incidents such as overheating, flat tires, and cars running out of fuel are common, and although these need to be cleared in a safe and timely manner, this is a greater challenger for highway first respondents when contraflow operations are in place. The authors highlight the need for developing intelligent transportation systems that can timely assess the impact of lane closures, weather conditions, and incidents to effectively guide evacuees.

Zou et al. [5] developed a simulation-based model for hurricane evacuations in the vicinity of Ocean City, MD. They

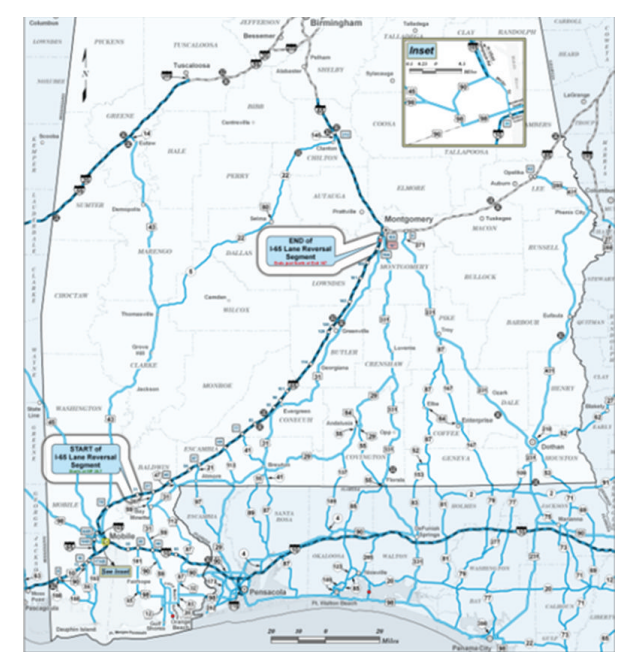

FIgURE 1: Alabama's I-65 contraflow evacuation plan.

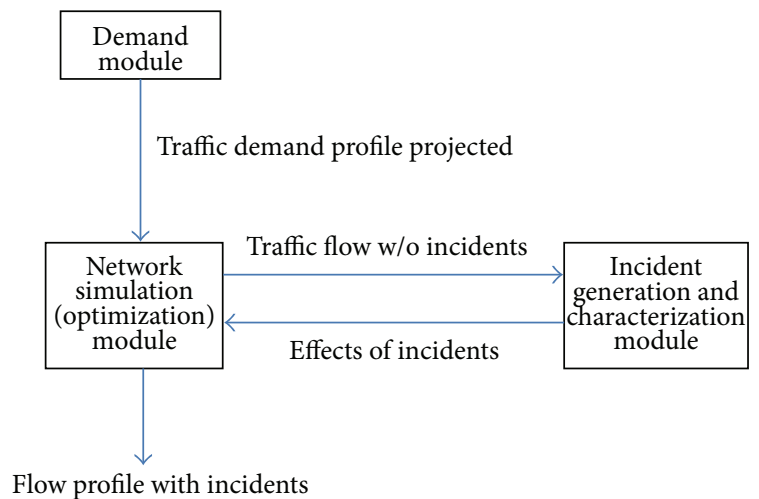

FIgURE 2: Contraflow DSS architecture.

devised six evacuation plans that involved real-time operational strategies during the simulated period of evacuation. One complication emanating from these real-time traffic operations was predicting travelers' responses under emergency evacuation. An embedded microscopic simulation engine was used by the authors of the study to assess traffic conditions under different demand patterns. This engine was developed with CORSIM, corridor simulation software by the FHWA, and involved a $2 \mathrm{~h}$ period of evacuation operations. The developed model allows users to investigate traffic conditions at critical points in the network under several control operations and potential incident scenarios. However, it is not clear how incidents are generated in the simulation, nor the extent or nature of the simulated incident scenarios.

Edara et al. [6] from the University of Missouri developed a large-scale traffic simulation model in VISSIM, a microscopic, time-step simulator, to estimate the performance of different evacuation routes, identify major sources of congestion, assess the overall network evacuation time, and devise traffic control strategies. Among the objectives of their research work was the simulation of incident occurrences to evaluate their impact on evacuation times. Incidents were 
randomly generated on high traffic volume segments through the use of the "partial routing decision" feature of VISSIM. That is, vehicular incidents were modeled as alternate routes for a time period within the time in which the static predefined traffic routing is in effect. Although this work represents one of the few serious efforts to introduce traffic incidents into massive evacuation analysis under contraflow operations, the way chosen to do so might not be appropriate for more complex, real-life situations due to its overall simplicity at assessing time delays and complete absence of congestion considerations.

Among the open literature, the work by Robinson et al. [7] can be singled out as the most comprehensive effort in studying the role of traffic incidents during hurricane evacuations. Through dynamic transportation simulations, the authors studied the impact of traffic incidents in Hampton Roads during emergency evacuations. The research involved simulations of evacuation traffic over a 70-hour period, and averaged almost 200 vehicular accidents and 1400 disabled and abandoned vehicles. The simulated scenarios were extracted from traffic databases, and the incident locations, severities, and durations were randomly selected from the available traffic data and match historical values. Even though this particular work does not consider contraflow operations, several of the assumptions and findings made by Robinson et al. [7] are very relevant to our work, and thus, they were used in the development of the incident occurrence and characterization module for Alabama's I-65 corridor.

\section{Incident Generation and Characterization during Hurricane Evacuation}

As depicted in Figure 2, the incident generator and characterization module interacts with the optimization module by triggering traffic incidents based on selected traffic counts and then temporarily reducing the flow capacities of road segments in the vicinity of the generated incidents. In essence, once the module has been activated as a "background routine" within the network simulation, it iterates every 60 minutes of simulated time executing the stochastic generation of incidents and characterizing them based on their duration and impact on the overall capacity of the evacuation network.

The processing of the module was designed and implemented based on traffic data, algorithmic procedures, and heuristics from ALDOT's evacuation logs of past hurricane events (Ivan and Dennis in particular), Alabama's CARE accident database, the Highway Capacity Manual [8], and related works by Qin and Smith [9], Zou et al. [5], and Robinson et al. [7].

3.1. Incident Generation. Incidents are generated based on the average traffic volume along the I-65 corridor and the time of the day. Accident logs as well as traffic counts during evacuation events were made available to the researchers by ALDOT for Tropical Storm Helen (September 2000), Tropical Storm Hanna (2002), Hurricane Ivan (September 2004), Tropical Storm Arlene (June 2005), Hurricane Dennis
(July 2005), Hurricane Katrina (August 2005), Hurricane Gustav (August 2008), and Hurricane Ike (September 2008).

As previously described, the section of Alabama's I-65 involved in contraflow operations is approximately 140 miles long, and it extends between exit 31 and exit 167 (refer to Figure 1). While generating traffic incidents within the 60minute window of simulated time, three decision variables needed to be determined: the number of incidents, their locations, and time of occurrence within the time window.

The available storm and hurricane evacuation logs did not provide any significant insights about particular segments of the I-65 corridor being more prone to traffic incidents than others. The only real relevant information obtained from the logs was the fact that all incidents took place between mile posts 37 and 167. Thus, it was established that for a given generated traffic incident, its location was to be defined according to a uniform distribution between miles 37 and 167. In other words, the probability for any traffic incident to take place at a particular location between these two mile posts is the same.

Regarding the number of incidents and their occurrence times, probabilistic distributions were developed using the traffic data recoded in the mentioned ALDOT's evacuation logs to model the incidents' interarrival times. The main parameters used to fit the stochastic distributions were the time of the day when the incidents were reported and the hourly traffic volume conditions measured as the average count for hourly volume readings at mile posts 43,87 , and 144 along I-65. The resulting incidents' interarrival scheme is depicted as follows (for instance, during an evacuation window between 8 a.m. and 9 a.m. when the traffic volume averaged above $1,000 \mathrm{vph}$, incidents are to be modeled according to an interarrival rate of $33+$ Weibull (10.1, 0.301) minutes).

\section{Interincident Times Distributions (min)}

From 4:00 a.m. to 1:00 p.m. and hourly traffic volume below $1,000 \mathrm{vph}$ :

Distribution: Lognormal,

Expression: $29.5+\operatorname{LOGN}(76,656)$,

Square Error: 0.272481.

From 1:00 p.m. to 8:00 p.m. and hourly traffic volume below 1,000 vph:

Distribution: Weibull, Expression: 33 + WEIB (10.1, 0.301), Square Error: 0.061510 .

From 4:00 a.m. to 1:00 p.m. and hourly traffic volume above 1,000 vph:

Distribution: Weibull, Expression: 9 + WEIB (23.1, 0.394), Square Error: 0.064422. 
From 1:00 p.m. to 8:00 p.m. and hourly traffic volume above 1,000 vph:

\author{
Distribution: Beta, \\ Expression: $32.5+48 *$ BETA $(0.349,0.288)$, \\ Square Error: 0.180530 .
}

${ }^{*}$ Hourly traffic volume is average of volumes at MP 43, MP 87, and MP 144.

It is important to notice that due to safety reasons, mandatory evacuation of residents along Alabama's coastal areas takes place between the early morning hours through sunset.

3.2. Incident Characterization. Once incidents are generated, they need to be characterized to assess their potential impact. Based on the Highway Capacity Manual [8], and the research conducted by Qin and Smith [9], Zou et al. [5], and Robinson et al. [7], it was decided to categorize traffic incidents into three different classes: (1) crashes, (2) abandoned vehicles, and (3) disabled vehicles. A crash happens when a vehicle exiting the evacuated zone collides with an object. This includes hitting a road rail, another vehicle, or crashing into a ditch. Abandoned and disabled vehicles are vehicles that for one reason or another stop moving. The difference between them is that an abandoned vehicle stops under the driver's control, as in the case of a flat tire or a nondisabled mechanical problem; hence the driver is normally able to pull the vehicle to the shoulder of the road. On the other hand, a disabled vehicle stalls with no imminent warning, which may result in a complete obstruction of a traffic lane.

Following the findings made by Robinson et al. [7] and through an extensive examination of the available hurricane evacuation logs (particularly those from hurricanes Ivan and Dennis) as well as Alabama's CARE accident database, it was concluded that out of all traffic incidents precipitated during a mandatory hurricane evacuation along I-65, 11.7\% can be classified as crashes, $4.8 \%$ as abandoned vehicles, and the remaining $83.4 \%$ as disabled vehicles. Thus, these ratios were used in this study to categorize the simulated traffic incidents.

The second phase of the incident characterization process deals with determining the effect that an already classified incident has on the evacuation network. In the specific case of Alabama's I-65, which is mainly a two-lane highway in each direction of traffic (i.e., north and south), an incident might affect the shoulder, one lane, or-in the most serious events-the two lanes in one direction. Based on incident simulations performed by Robinson et al. [7], as well as the investigators' own observations, the percentages depicted in Table 1 were established to quantify how incidents such as crashes, abandoned vehicles, and disabled vehicles obstruct the free flow of traffic along Alabama's I-65. For instance, it was determined that ninety percent of disabled vehicles only impact the shoulder of the freeway while the remaining ten percent will indeed obstruct traffic in one of the two lanes of the highway.

Finally, once incidents have been generated, categorized, and their impact on the roadway (i.e., I-65) assessed,
TABLE 1: Traffic incidents effects during hurricane evacuations along I-65.

\begin{tabular}{lccc}
\hline & Shoulder & One lane & Two lanes \\
\hline Crash & $75 \%$ & $18 \%$ & $7 \%$ \\
Abandoned vehicle & $100 \%$ & $0 \%$ & $0 \%$ \\
Disabled vehicle & $90 \%$ & $10 \%$ & $0 \%$ \\
\hline
\end{tabular}

TABLE 2: Altered road capacities by incident type.

\begin{tabular}{lcc}
\hline & Blocked & Capacity available \\
\hline \multirow{3}{*}{ Crash } & Shoulder & $81 \%$ \\
& One lane & $35 \%$ \\
& Two lanes & $0 \%$ \\
\hline Abandonment & Shoulder & $95 \%$ \\
\hline \multirow{2}{*}{ Disablement } & Shoulder & $95 \%$ \\
& One lane & $35 \%$ \\
\hline
\end{tabular}

the incident detection and characterization module computes the reduction in road capacity at the location of occurrence. The way the module does this is by altering the default capacity in the network simulation module for the road segment stretching a mile north and a mile south (i.e., \pm one mile) from the location of the incident. The altered road segment capacity stays in effect for an interval of time equal to the duration of the incident. Table 2 shows the altered road capacities for the different incident scenarios. For example, as seen in Table 2, a crash that only affects the road shoulder will imply a $19 \%$ reduction in the capacity of the road section within two miles of its location. If the crash obstructs one lane of traffic, the capacity for the affected road segment will be reduced to thirty-five percent of its original capacity or zero if both lanes are blocked.

Table 3 depicts the duration distributions for the generated incidents. For instance, it is assumed that $70.5 \%$ of all disabled and abandoned vehicles will be resolved (that is, cleared) within 15 minutes of their occurrence and $18.4 \%$ of such events will take between 15 and 30 minutes to clear, while the remaining $11.2 \%$ may take somewhere between 30 and 60 minutes. These incident durations as well as the estimated road capacity reductions shown in Table 2 were established after a comprehensive study that involved detailed analysis of ALDOT's hurricane evacuation logs, as well as adaptation of the approaches followed by Qin and Smith [9], Zou et al. [5], and Robinson et al. [7] originated from the Highway Capacity Manual [8].

\section{Implementation}

A proof-of-concept of this design was constructed using Excel and evaluated using historical data. When activated, the incident generation and characterization module provides the network simulation module with the times of occurrence and locations of traffic incidents generated within succeeding 60 -minute windows of simulated evacuation time. The precipitation of a particular incident leads to a predetermined road capacity reduction at the impacted location (i.e., within 
TABLE 3: Incident duration distributions.

\begin{tabular}{lcc}
\hline Type of incident & Ratio & Duration $(\mathrm{min})$ \\
\hline \multirow{3}{*}{ Disabled/abandoned } & $70.5 \%$ & Uniform $(0,15)$ \\
& $18.4 \%$ & Uniform $(15,30)$ \\
& $11.2 \%$ & Uniform $(30,60)$ \\
\hline \multirow{3}{*}{ Accident/crash } & $23.5 \%$ & Uniform $(0,15)$ \\
& $20.1 \%$ & Uniform $(15,30)$ \\
& $56.4 \%$ & Uniform $(30,60)$ \\
\hline
\end{tabular}

TABLE 4: Total system travel times of incident scenarios (in pcu.hr).

\begin{tabular}{lcc}
\hline Number of run & \multicolumn{2}{c}{ Total system travel time } \\
& Low demand & High demand \\
\hline 1 & 180220 & 1494479 \\
2 & 181431 & 1498361 \\
3 & 180222 & 1493513 \\
4 & 180220 & 1490705 \\
5 & 180220 & 1491812 \\
\hline
\end{tabular}

a two-mile stretch) for the time it takes for the incident to clear. Incidents were then generated for two different traffic scenarios, that is, low and high demands.

In the low demand scenario, a category-3 hurricane was simulated with a total evacuation demand of 64,568 passenger car units (pcu). The high demand scenario involved 152,400 pcu evacuating the region due to a category-5 hurricane. A total of 48 and 104 incidents were generated for each of the scenarios, respectively. Table 4 shows the total amount of time spent by all the vehicles on I-65 under these demand scenarios when incidents were simulated. Each scenario was simulated five times (i.e., each simulation run with a different random seed).

The mean total time spent by all vehicles was $180,463 \mathrm{pcu} \cdot \mathrm{hr}$ and $1,493,774 \mathrm{pcu} \cdot \mathrm{hr}$ under the low and high demand scenarios, respectively. Thus, we can roughly say that under low demand, the average time a vehicle spent on Alabama's I-65 during an evacuation event is $2.79 \mathrm{hrs}$. This average time increases to 9.80 hours under extreme evacuation circumstances (i.e., considerably high demand of vehicles trying to exit the region).

When these scenarios were run without incidents, the average total travel times obtained were 180,220 pcu.hr and $1,492,402 \mathrm{pcu} \cdot \mathrm{hr}$ for low and high demands, respectively. Consequently, when assessing the impact that incidents have on the total time spent by all vehicles on I-65 during hurricane evacuations, we can say that the total travel time increases, on average, by $243 \mathrm{pcu} \cdot \mathrm{hr}$ when there is a low volume of vehicles evacuating the area and $1,372 \mathrm{pcu} \cdot \mathrm{hr}$ when a high traffic volume demand is present.

\section{Conclusion and Remarks}

This paper described the development of an incident generation and characterization module incorporated into a decision support system environment for the assessment and optimization of contraflow operations during hurricane evacuation along Alabama's I-65 corridor. The developed module was designed and implemented based on traffic data, algorithmic procedures, and heuristics from ALDOT's evacuation logs of past hurricane events, Alabama's CARE accident database, the Highway Capacity Manual [8], and related works by Qin and Smith [9], Zou et al. [5], and Robinson et al. [7].

This work represents one of the few reported attempts to investigate and assess the impact of traffic incidents on the overall evacuation travel time of vehicles exiting the affected area. As conventionally defined, incidents are nonrecurrent events such as crashes, abandoned vehicles, and disabled cars that, under oversaturation conditions, can represent a considerable source of congestion.

The designed incident generation and characterization methodology was eventually implemented under two different evacuation scenarios. The first one when a low demand of evacuation traffic was assumed and the other when a high flux of departing traffic was present. The results showed that nonrecurring incidents are more a safety factor than a flowdisrupting one for controlled, mandatory evacuation events. It was observed that the percentage increase in total travel time due to incidents was only $0.13 \%$ for low demand and $0.09 \%$ for high demand. This result suggests that the concerns that are usually articulated about traffic incidents during evacuations are misdirected.

\section{Acknowledgments}

Special thanks are extended to the Alabama Department of Transportation (ALDOT) for funding this effort. The authors also wish to thank George Connors and Stacey Glass for their efforts and assistance with this project.

\section{References}

[1] Alabama Department of Transportation, "I-65 Contra-flow Plan: 2010 Hurricane Season,” 2010, http://ema.alabama.gov/ filelibrary/Alabama_EOP.pdf.

[2] B. Wolshon, “'One-way-out': contraflow freeway operation for hurricane evacuation," Natural Hazards Review, vol. 2, no. 1, pp. 105-112, 2001.

[3] M. K. Lindell and C. S. Prater, "Critical behavioral assumptions in evacuation time estimate analysis for private vehicles: examples from hurricane research and planning," Journal of Urban Planning and Development, vol. 133, no. 1, pp. 18-29, 2007.

[4] B. Wolshon, E. U. Hamilton, M. Levitan, and C. Wilmot, "Review of policies and practices for Hurricane evacuation. II: traffic operations, management, and control," Natural Hazards Review, vol. 6, no. 3, pp. 143-161, 2005.

[5] N. Zou, S.-T. Yen, G.-L. Chang, A. Marquess, and M. Zezeski, "Simulation-based emergency evacuation system for Ocean City, Maryland, during hurricanes," Transportation Research Record, no. 1922, pp. 138-148, 2005.

[6] P. Edara, S. Sharma, and C. McGhee, "Development of a large-scale traffic simulation model for hurricane evacuationmethodology and lessons learned," Natural Hazards Review, vol. 11, no. 4, pp. 127-139, 2010.

[7] R. M. Robinson, A. Khattak, J. A. Sokolowski, P. Foytik, and X. Wang, "What is the role of traffic incidents in hampton roads 
hurricane evacuations?" in Proceedings of the Transportation Research Board Annual Meeting (TRB '09), pp. 1-17, 2009, CDROM.

[8] Transportation Research Board, Highway Capacity Manual, National Academy of Sciences, Washington, DC, USA, 4th edition, 2010.

[9] L. Qin and B. Smith, "Characterization of accident capacity reduction," Research Report UVACTS-15-0-48, Center for Transportation Studies, The University of Virginia, 2001. 

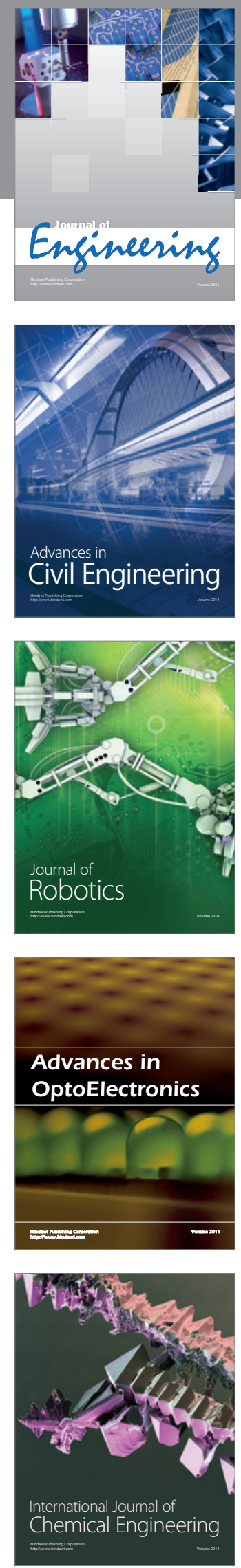

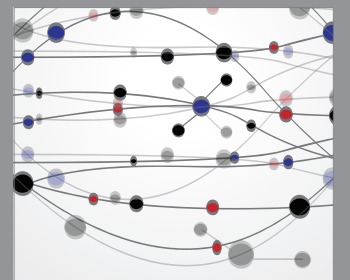

The Scientific World Journal
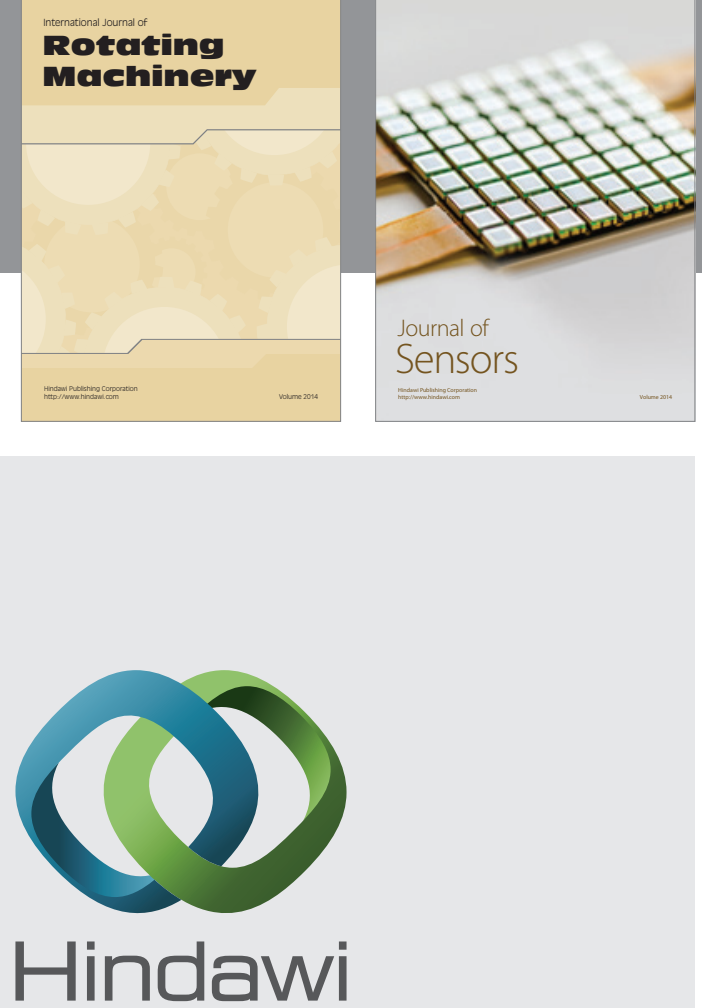

Submit your manuscripts at http://www.hindawi.com
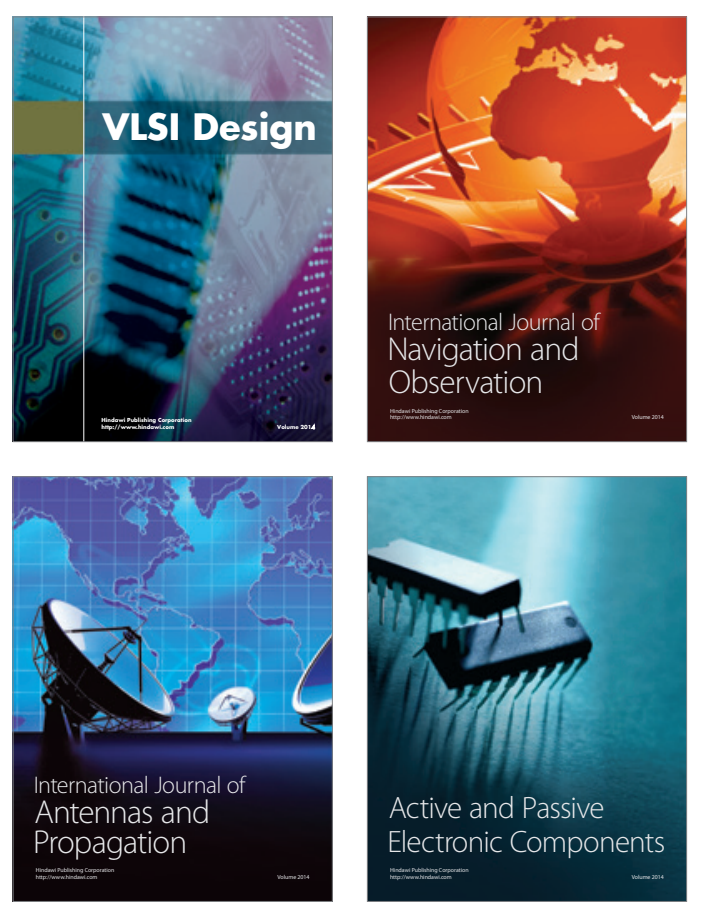
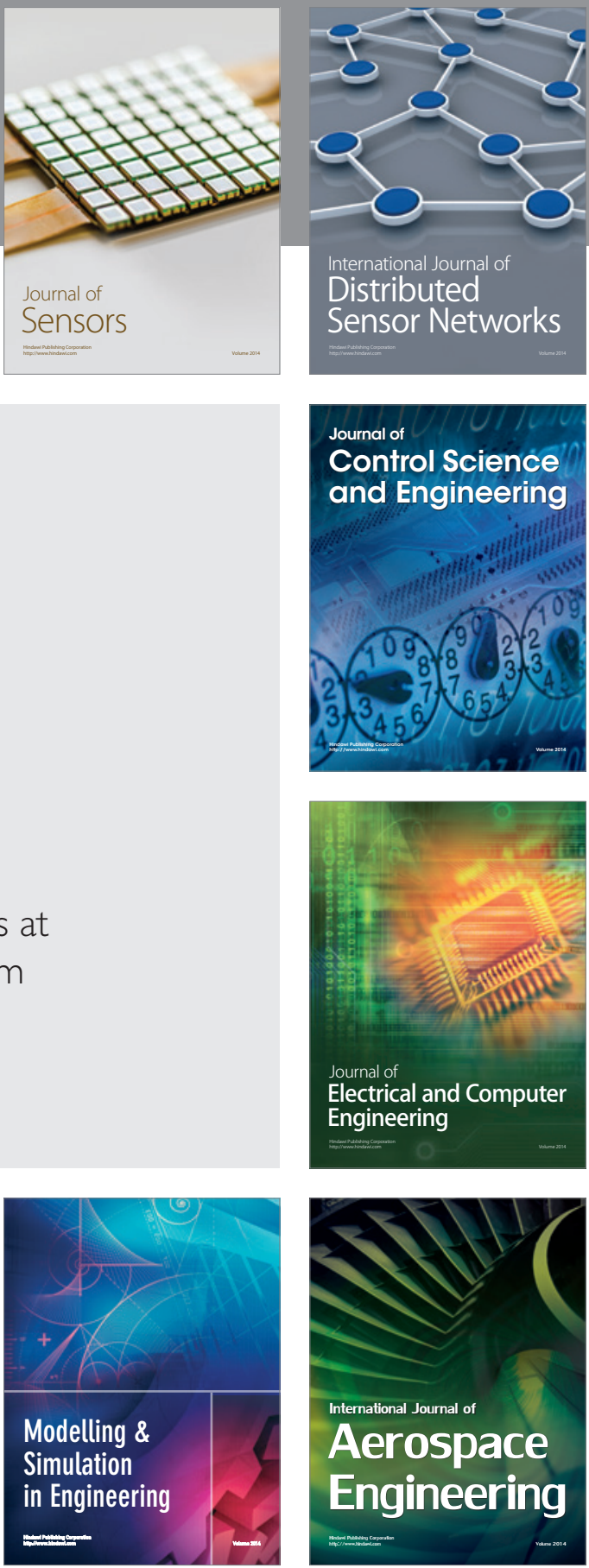

Journal of

Control Science

and Engineering
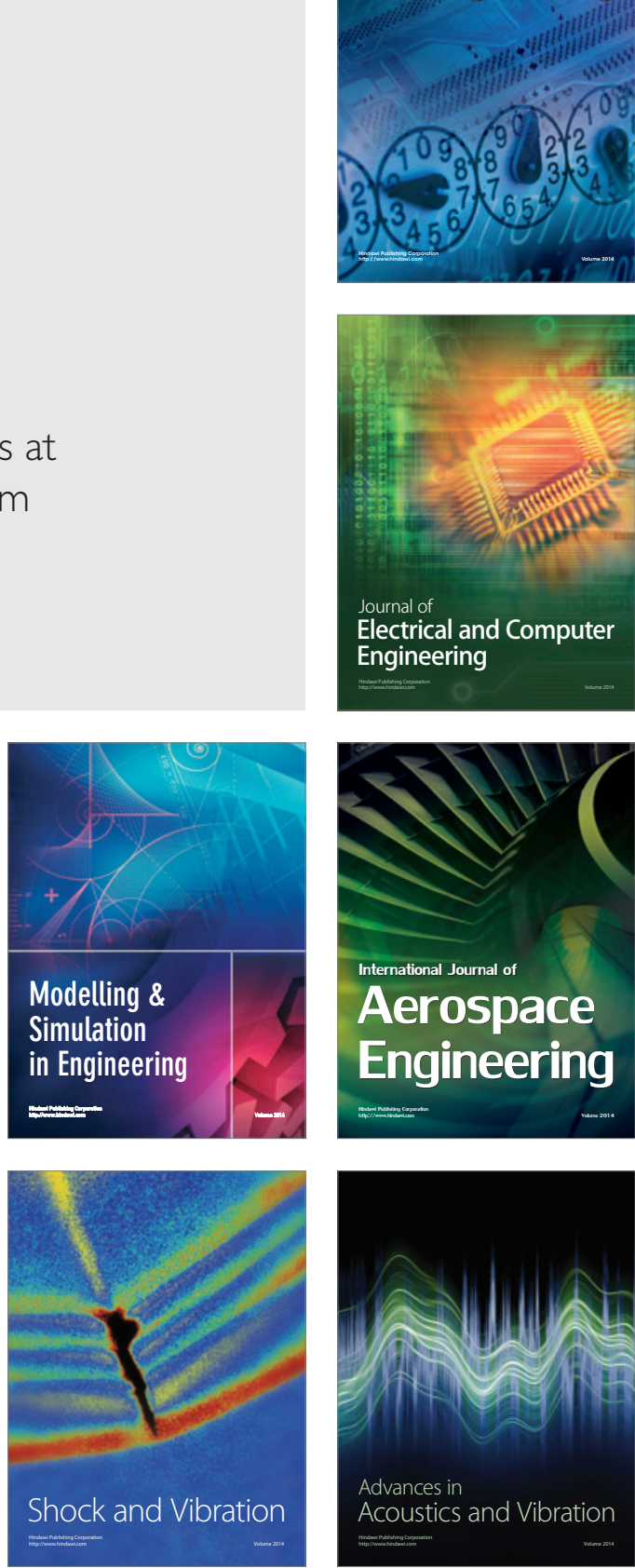